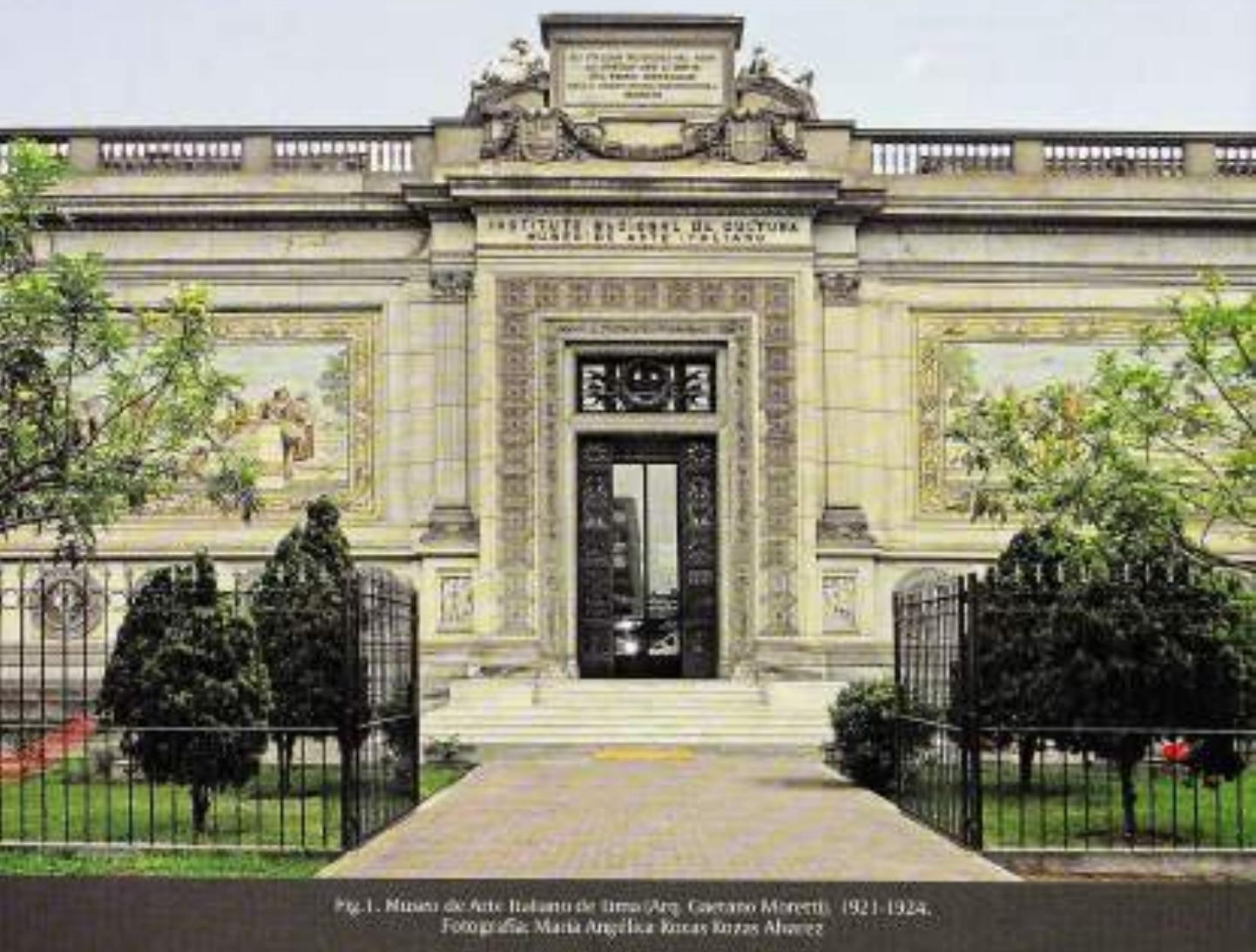




\section{Un obsequio muy peculiar: el Museo de Arte Italiano de Lima*}

Enzo Barsefiño Universided Roow 3

El autor conoció el Museo de Arte Italiano de Lima en 1987 cuando fue invitado para dictar unas clases sobre la arquitectura barroca y se quedo miy sorprendida al encontrar un museo pequeño pero tan interesante, que conservaba obras de artistas italianos de los años 20, seleccionadas para ser regaladas por los italianos que vwiar en el Perve en ese entonces en ccasibn delprimer centenario de la independencia del pais |1921\}.

Esta contribución, aue traza brevemente la histora del museo, guiere volver a proponer la Idea de una exposción que se or ganice primero en Lima y loego en Rcma (con una selección de obras\} para mestrar lo valioso que es el singular y único museo donado por los italianos al Porú.

Museo de arte / Arte italiano / Comunidad italiana / Siglo XX / Centenario de la Independencia del Perú

The author knew the Museum of Italian Art in Lima in 1987 when he was invited to Lima to pive some lessons on Raman Baroque architecture. He was very surgrised to find a small but Interesting museurn which collected works of Italian artists active in the 20 s of the Twentieth Century. These were selected to be given as gifts by the Italians who lived then in Peru in 1921 on the occasion of the first centenary of the independence of Peru.

This paper, tracing briefly the history of the museum wants to revive the idea af the exhibition to be organized prior in Lima and then in Rome (with a chcice selecton of works) to give a contribution to the enhacement of this uniqua and singular museum donated by the Italians to Pers.

\section{Art museum / Italian art / Italian community / Twentieth Century / Peruvian Independence Centerary}

Tradacción: Grazia lesta

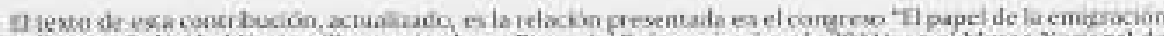

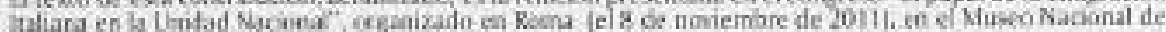

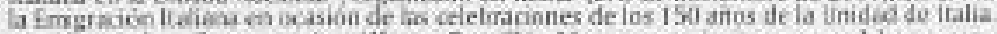

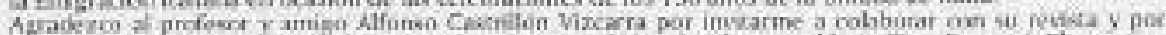

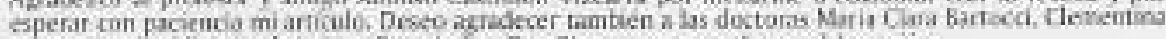

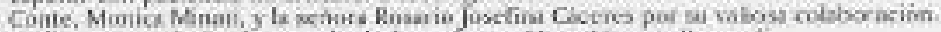

Dedios este aticulo al recuerdo de lo prefesora Mara vitrata Hugroli. 


\section{Prólogo}

Mi primera visita al Museo de Arte Italiano de Lima fue ya bace muchos años 1987 ), durance un inohidable viaje al Perí que empezó justamente en Lima. En la capital peruana, invitado por los profesores Afonso Castrillon Vizcarra y Renato Lipari, dicte algunas clases sobse la primera arquitectura barroca tomana en el instituto Italiano de Culura de dicha ciudad. ISübitanente me quedé fascinadol Nunca habrit imeginado ver en otro continente una colección de arte italano de comienzos de 1900 tan pecaliar y tan significativa.

Cono estudioso de histonia del coleccionisme y de los museos en segtida me apasione por ef tema, además porque la caracteristica más notable y ha unidad de esta institución son el hecho de representar un regalo de la comusidad italiana es ese entonces presente en el Pení para ceiebrar kos primeros cien aivos de la incependencia de la nación sudamericana (1921). I as comonidades de los demas paises habian ofrecide una fuente crmamental un monumento, un enorme reloj, pero nadie nunca, para una ocasión similar, habia pensado regalar un musen entero.

De regreso a Italia empecé la buisqueda de noticias y documentos refurentes al Museo de Arte Italiano consultando el archivo histócico del Ministerio de Relaciones Exteriores donde persaba podrian estar conservados escritos y cartas sobre el tema.

Los documentos encontrados y la literatura poblicada hasta ese momento, empezando por el catalogo de las obras conservadas en el maseo, editado por Uzo Ojetti en italiano y en espaniol', me remitieron en seguida a otro fondo de archivo que me podria proporcionar mass informaciones: el Fondo Ojetti, depositado en el Archivo Histórico de la Galeria Nadional de Arte Moderno y Contemporaneo, Desafortunadanente en aquel entonces el fondo un se podía consultar, y no se pudo hexcer durante muchos ahos mas, asi que, como a menudo pasa, presicaado por otras buisquedas, abandoné el proyecto.

En 1994, inesperadamente para m, se publico un nuevo catalogo del Museo de Lima, a cargo de Mario Quesada, gracias al renovado interés hacia el museo, mativado en esa epoca por el embajador de Italia en el Perú Bemarchino Osio. Se trató de una importante actualización cientifica sobre las obras del museo, pero, ai siquiera en aquella ucasión, se recanstruyo por completo la fascirante aventura de Mario Vannini Parenti y de los otros miembros del comité que realizó esta singular hazaña.

En 2010, el profesor Augusto Ferrero, entonces embajador del Perú en Italia, llevō a mi universidad 'Roma Tre' la propuesta de traer a Roma una selección de las obras del Museo de Arte Italiano de Lima para exponerlas. y pareció haber llegado el momento de terminar mis busquedas. El Fondo Ojeffi del archivo histórico de la Galeria de Arte Moderno y Contemporineo ya se podia consul tar, asi que empecé a leer los primeros fasciculos, sin embargo el proyecto no pudo seguir pot falta de presupatesto.

En noviembre de 2011 se organizó un congreso en Roma, con ocasión de las celebraciones de los 150 aíos de la Unidad de italia ([86]) en el Musco Nacional de la Emigracion Italiana cuyo tema era "El papel de la emigración Italiana en la Unidad Naconal" Ë momento me pareció oportuno para hablar publicamente del Museo de Arte Italiano cuya historia se relacionaba de manera essrecha con el tema del congreso y para volver a proponer la idea de la exposición.

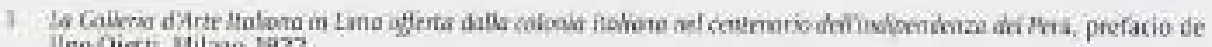
IIgo-gieti, Milane 1922

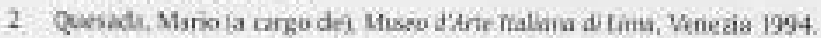

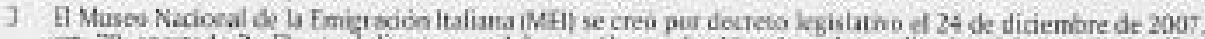

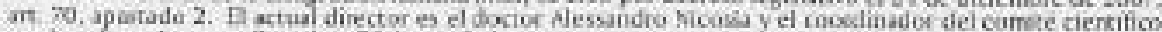

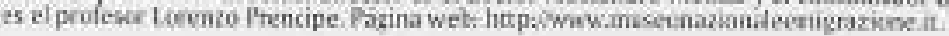




\section{Los italianos en el Perú}

Sobre la historia de la migración italiana al Perú es convenience hacer referencia en especifico a los textos de Manuel Zanutelli Rosas y de Giovanni Bonfiglio', entre oxros, y, sobre la emigracion en general hasa las Americas, a Ado Albonico y Gianfausto kosoli" Tambien

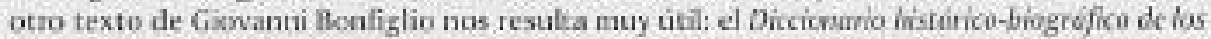
italianos en el Perì Como ed mismo Penafiglio afirma en su introducción:

\begin{tabular}{|c|}
\hline $\begin{array}{l}15 \\
\text { ay } \\
\text { tâ } \\
70\end{array}$ \\
\hline
\end{tabular}

La comanidad italiana está presente en el Peru desde la eponca de la colonia y los primeros italianos erat sobre todo comerciantes y marineros. Más tarde encontramos artesanos; artistas y religiosos. $5 \mathrm{egua} \mathrm{lo} \mathrm{que} \mathrm{escribió} \mathrm{Luigi} \mathrm{Guarnieri} \mathrm{Calò} \mathrm{Carducci} \mathrm{en} \mathrm{la} \mathrm{presentación}$ del Diccurnorio de Bonfiglo y al que remito para los dasos citados en el presente articulo:

1. La inmigración italiana en el Peri de cierta importancia empieza poco después de la independencia del pais, en la tercera década de 1800. EI flujo se debe todavia a razones maritimas y comerciales. A partir de 1840 la presencia icaliana, manomente de marixeros tigures, empieza a diferenciarse, con un moderado componente de agncultores de profesión $y$ de prólugos por razones politicas" Analizamos un poco los numeros.

En 1876 en Peru habia siete mil italianos, cae bajaron a seis mil en 1900 . En 1940 eran menos de cuatro mil. Las cinco mil sctecienras presencias después del eanflicto mundial atestiguas cỏno la inmigracion se tuanudó notablemente. En las siguientes décadas vohvici a bajar: en 1971 cinco mit. en 1980 poco menos de ctutro mil. La comunidad italiana màs mumerosa está en lima, con un porcentaje promedio, enire 1900 y 1981 de 600 y $78 \%$ en total, seguida por la del Callao. Comparados con los demás europeos, los italianos constimyen la comunidad inas considerable hasta los anos '70 de 1900 . Mientras que en los otrus paises tatinoanericanos los italianos liegaban de todas las regiones de italia, aunque en diferentes proporciones, en el Peru siguen siendo preponderantes los ligures. Las caracteristicas de esta emigración regicnal han pernitido un flujo de regreso a las localidades de origen. que contriasaron reviendo estrechos contactos con los que se habian quedado en Peri. Guarnicri Calo Carducci reclerda que hasta hoy en Chiavari se celebra la fiesta nacional peruana del 28 de julio.

\section{4}

3

6

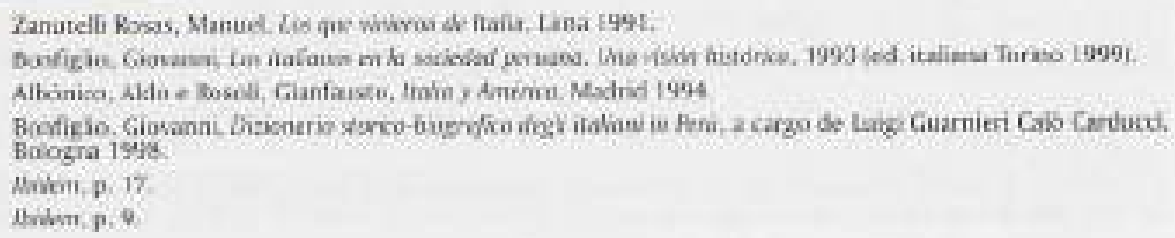


En 1800. Ios italianos eran el 4 v de la población de la capital del Perú a finakes de los años 90 de 1900 el porcentaie erd mucho menor, debajo del $1 \%$ por otro lado, en la misma epoca, seguin los datos de la Embajada lialiana de Lima, fabia aproximadanente 16.000 persinas con ciudadania italiana. De ellos, te mayoria. altededor del rok, habia nacido en el Perú. La disminución de las Begadas y la adaptación progreciva a la saciedad peruana. gracias tambien a los matrimonios mixtos, han contribuido a la integración social y cultural de los descendientes. que llegaron ya, en algumos cassos, a la quinta generación y a los niveles más altos de la sociedad peruana.

La inmigracion italiana en el Perii se caracterizó por flujos regulados en base a las posibilidades de asimilación, sobre todo en el sector maritimo comencial, primero local y luego hasta intemacional: lpensemos por ejemplo en la cra del guano! $\gamma$, despues, desde la cos. ta. las actividades comerciales se desartollaron hacia el interior, como en el caso del eje Lomas-Cuzco y el de Mollendo-Puno. En el sector agricola, por otro lado, hubo reiterados fracasos, en especial en las plantaciones y en la colonización de territorios virgenes.

Frente 2 la inestabilidad politica pertana de 1800 y a la inseguridad social, los italianos fundaron varias instituciones de socorre mutuo, asistencia, solidaridad, que han tenido tambien una función de refuerzo en las relaciones culturales y sociales. Sir umbargo en 1900 las instituciones italianas perdieton su funcion de protección frente a la inestabilidad de la sociedad peruana y conservaron la función de referencia culfural, ya que los inmigrantes italianos y sus descendientes habian entrado en las sectores medio-altos de la sociedad:

...Asi la caracteristica principal de la presencia italiana -recuerda una vez más Guarnieri Calo Guarducci-, siempre a la vanguardia con su espiritu proactivo y su capacidad de adapxación. fue la diversificación de las actividades empresariales en los sectores más dinámicos; er especial, además del sector comercial, en el sector de la industria, con plantas para trabajar el algodón, el azucar, los tejidos, los alimentos, y en el sector de los servicios. Con respecto a este ültimo ámbito. resulta de impostancia primordial el concurso italiano para constituir una entidad finabciers y bancaria" " pensamos pox ejemplo en la constitucion del Banco Italiano, fuadado por emprestrios italianos de los que vamos a hablar más adelante.

A parte de la actividad cconónica. los italianos desempenaron y desempenan todavia hoy en dia un papel relerante en las instituciones politicas, económicas y culturales, ocupando tambien cargos significativos. I.os italo peruanog tuvieron frectentemence funciones relacionadas con el desamallo de la educación y de la cultura. en escuelas y museos, ademas de un rol muy apreciado en el ámbito artistico y masical.

\section{Mario Vannini Parenti, Ugo Ojetti y el Museo de Arte ltaliano en Lima}

El Diecionario de Bonfiglio antes mencionado registra indirectamente en afgunas biogtafiss extensos rastros del evento de la fundación del Museo de Arte la liano a traves cle las entra. das referentes a: Giuseppe Boero, Giovanni Carosio, Gaetano Moretti, Egisto Novelli, Gino Salocchi, Mario Vannini Parenti."

1. comunidad de los italianos residerstes en el Perú fundó en 1921 un Comité Italiano del Centenario cuyo presidente era el médico Giusepoe Boero. También Fgisto Novelli y

10 Unchin, $p, 12$

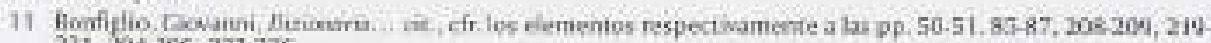
221. $294 \cdot 296.333-335$. 


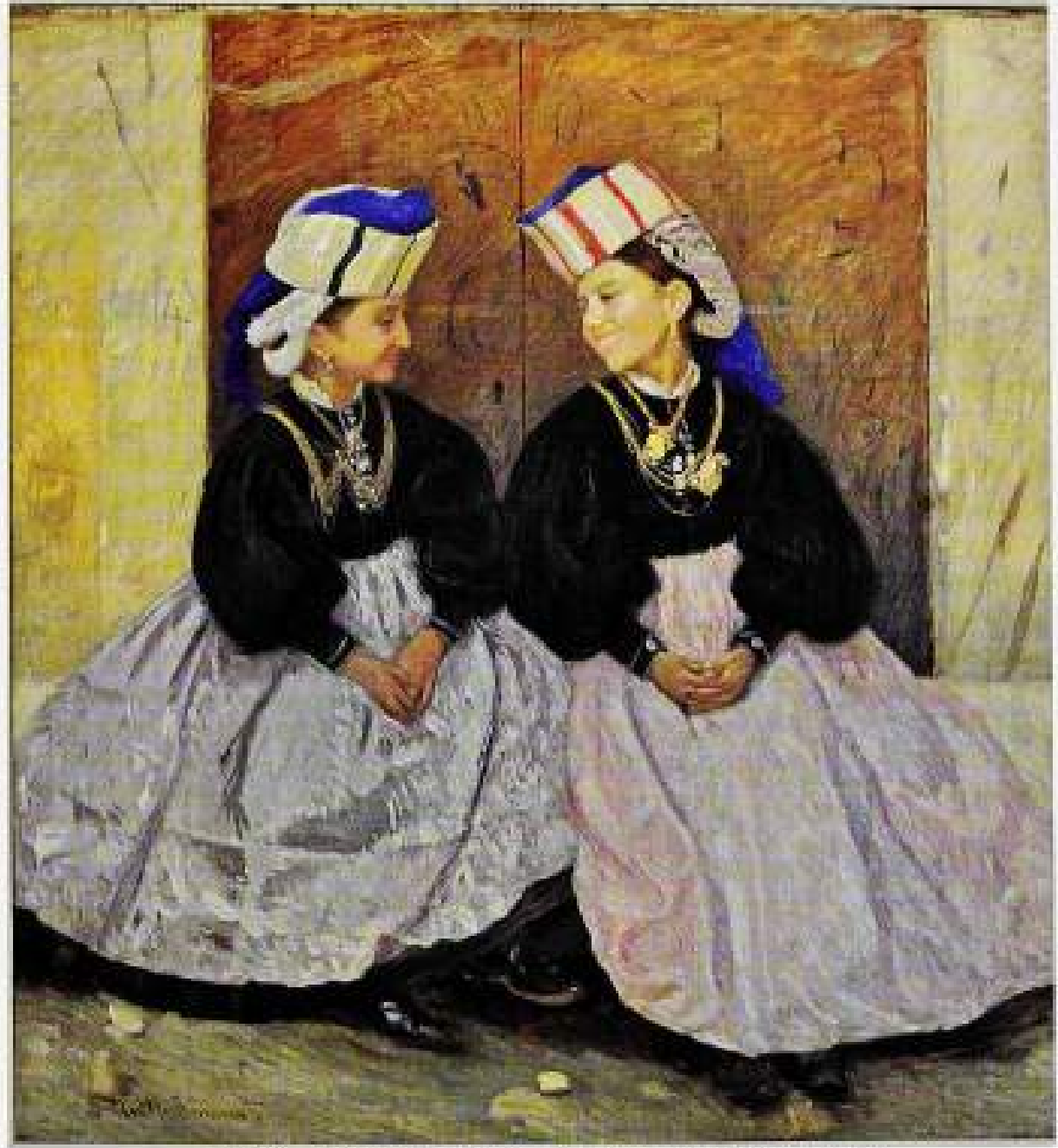

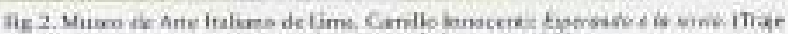

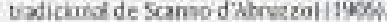

Giovanni Carosio dieron su contribución. Todos colaboranon con su peculio para ofrecer este presente que tertia un doble valor: mostrar gracitud bacia el puis que los acogia y al misno termpo proporcionar usa muestra del genio italiano que se basaba en una tradición de sizlos de extracordinaria produccion artisticat.

El protazonista de este singular proyecto fue el flocentimo Mario Vannini Parenti 11887. 1983! anticuario y conocedor de arte que yon habla dado a conocer obras de artistas italianos en Argentina y en otros paises sudamericanos, y al cual, por eso, se habia encargado la parte operativa feferente i la busqueda de las obras de arte. Sat-colabotador era Gino Salochi I 882-1951/ el entonces Director Ceneral del Ilanco Italiano, después Panco de Crédito, que contribuyb en primera persona y coordino la recaudacion de fondos entre los connacionales para la compta de los materiales artistices. El anticuario y el banquero se 
volvieron hego garte de la misma familia ya que el hijo de Vannini Parenti, Gianfranco, se caso con Nicoletta Salocchi, hija de Gino.'?

Se trata de los dos principales anianadores de una extraondinaria iniciativa que celebrara $\mathrm{f}$ soleme festividad de la nación perwana y demostrara la apreciación de los italianos con un acto sorprendente: ofrecer alrededor de doscientes obras de arte fealizadas por artistas italianos contemporáneos y un edificio donde hospedarlas en un area otorgada por la murecipalidad de Lima en el Paseo de far Fepublica, et Parque Nepuno. en esa epoca una $200 \mathrm{a}$ en plena expansion urbana hacia el sur de la ciudad.

Ugo Ojetrif1871-1946), muy conccido come critico de arte y de arquitectura' desde Ifalia brindó apowo con la lousqueda y con la selección de las ebsas por adquirir. Su objetive era ofrecer us panoraina de las diferentes tendencias attisticas presentes en las regiones italiamas. A su lado destacaban el escultor Piemo Canonica $(186 \%-1959)$, ef pintor Camillo Innocenti $\{1871.1961$, de los cuales el museo conserva algunas obras, y el Director Genera de Antiguedades y Bellas Artes del Mintisterio de Educaxión Pública de la época Arduino Colasanti (1877-1935). En realidad el Ministerio numca intervino avalando formalmente ta inichativa, sin embargo, en la docunentacion consultada se denuestra el interés y el apoyo de La máxima institución públice italiana que se ocupabe de la gestion del patrimonio his. tórico-artistico y de la producción artistict comterpoceánea. " De hecho el mismo Colasanti solicitó la formación de una cosusión para la recolección y la selección de las obras que se ban a enviar a lima. Ádemás de los artiba menconados era parte de dicha comision Gaetano Moretti 1860-1938), el arquitecto escogido para diseâar el edificio que alberyaria la colección. "Moretti habia desempenado muchos y exisosos cargos en Italia y en America Latina" y sus amistades en la colonia de ligures del Peru sin duda ka beneficlaton. Los proyectos arquitectónicos para el museo todavia no han sida bollados y, probablemente se han perdido. IT

La primera piedra de la construcción se puso el 30 de fulio de 1921 , en 1922 el edificio estaba terainado. La sociedad constuctora fue ta empresa italo-argentina Pob-Lavanes; dirigida por el ing. Montanati que en lama construyo tanhen la sede del Manco Italiano.

Una de las primeras descripciones del Museo de arte Itatiano, traxada par Ambrogio Aunoni," alumno y estrecho colaborador de Moretti, aciara la woluntad del proyectista

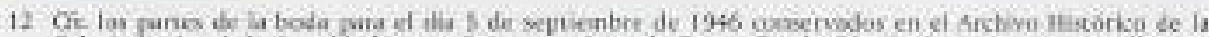

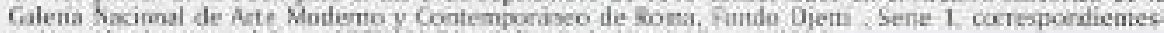

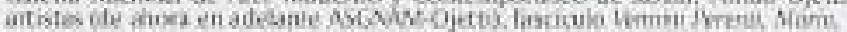

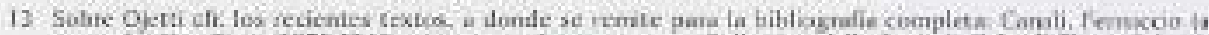

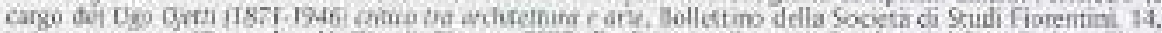

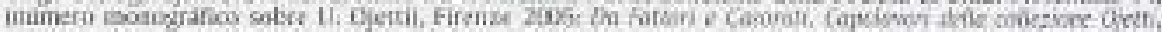

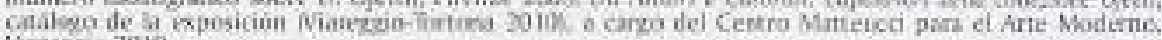
Yameze 2010

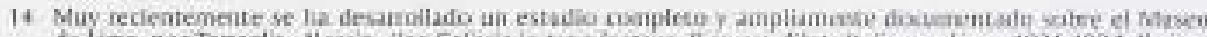

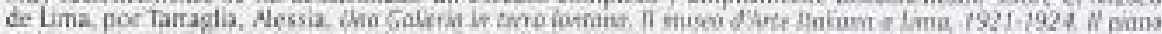

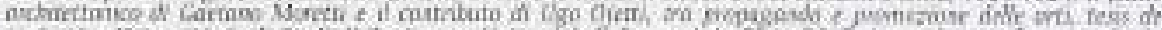

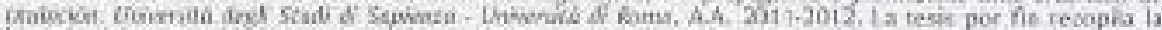

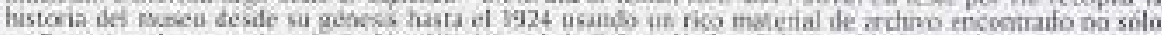

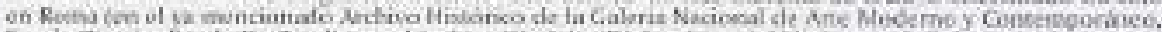

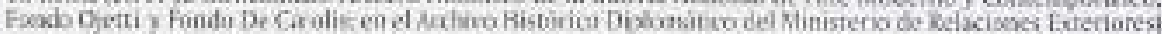

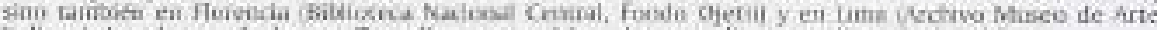

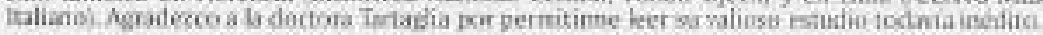

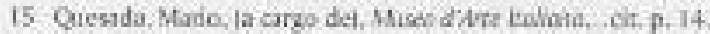

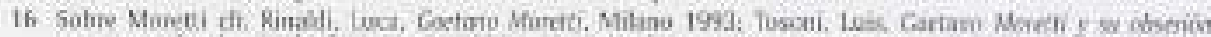

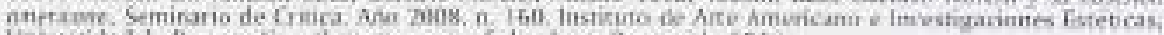

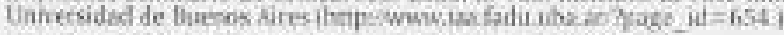

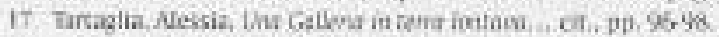

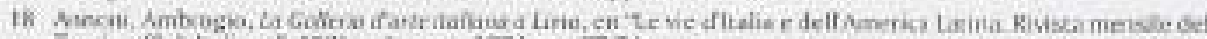

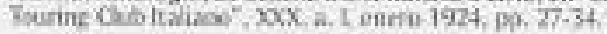




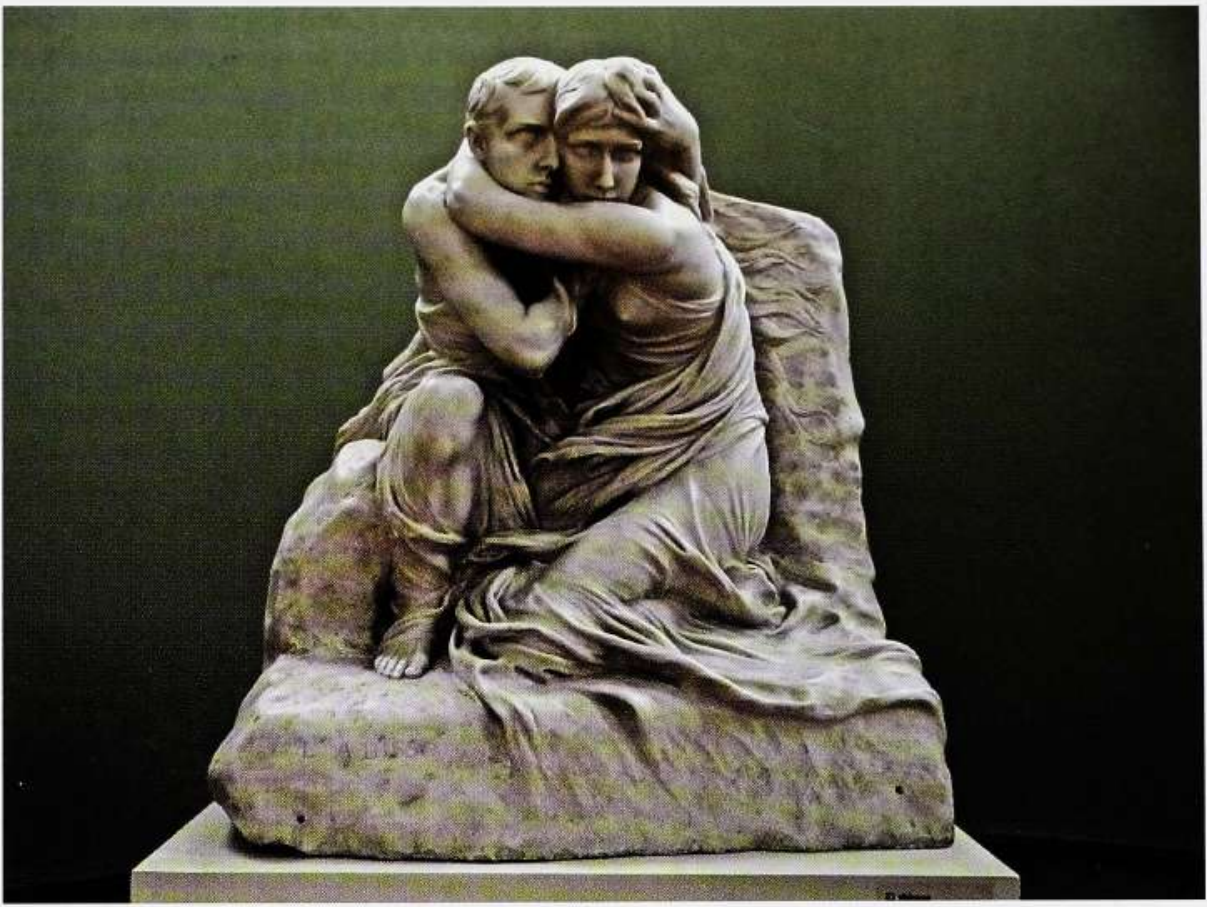

Fig.3. Museo de Arte Italiano de Lima. Pietro Canonica: El Abismo (1906). Fotografia: Maria Angélica Rozas Rozas Alvarez

de inspirarse -"sacrificando su propia personalidad"- en las formas más puras y famosas de la arquitectura italiana, seguramente también por pedido de los comitentes. ${ }^{19}$ Aunque el pabellón en su conjunto recuerda edificios expositivos parisinos de la misma época, entre el modernismo culto y el eclecticismo de 1800 (fig.1), para la decoración externa la inspiración fueron los grandes maestros del Renacimiento Italiano, sobre todo Bramante (el basamento externo con los emblemas de las principales ciudades italianas), Miguel Ángel (las copias de la Aurora y de la Noche en la coronación del portal de entrada), Donatello (los amorcillos danzantes a la base de los pilares angulares y a los lados del friso del portal de entrada) y otras referencias. Los mosaicos decorativos a los lados del portal de entrada confirman la misma línea de inspiración, representando "hombres ilustres" italianos. Es evidente la intención de mostrar el "genio italiano" en todas sus figuras: artistas, científicos, literatos, condotieros, héroes, exploradores, filósofos, inventores, en el marco neo-rafaelista que recuerda La Escuela de Atenas. Asimismo todo el museo "hablaba italiano", manifestando la gran habilidad de los artistas, artesanos y decoradores italianos herederos de un pasado glorioso del cual conseguían igualar la calidad. ${ }^{20}$ Hasta los tapices en seda de las paredes internas habían sido tejidos en Florencia y enviados a Lima.

19 Es el mismo Ojetti en la introdución del catálogo quien recuerda que Moretti tuvo que adaptarse a los requerimientos del comité (La galleria d'arte italiana.... cit., p. 1).

20 El milanés Alessandro Mazzucotelli (1865-1938) realizó el portal de entrada en hierro; el florentino Luig Lelli (activo en el siglo XX) reprodujo las dos alegorias de Miguel Angel: Giovanni Buffa (1871-1954) dibujó los mosaicos que después fueron ejecutados por una cooperativa de mosaiquistas venecianos: en el interior trabajaron en los frisos de las salas el florentino Salvino Tofanari (1881-1969), en los vitrales Ezio Giovannozzi (1882-1964) y Giovanni De Matteis (1852-1930). 


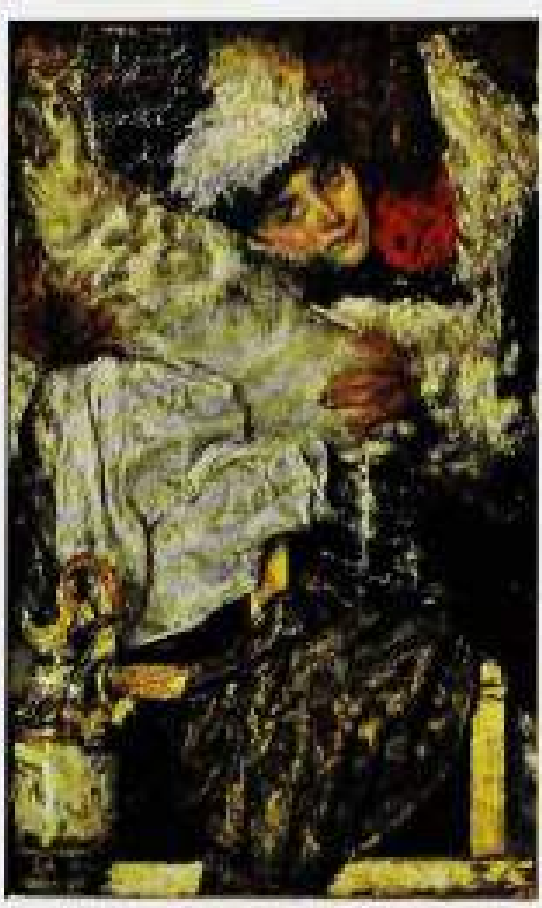

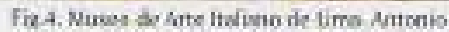

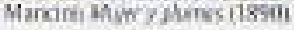

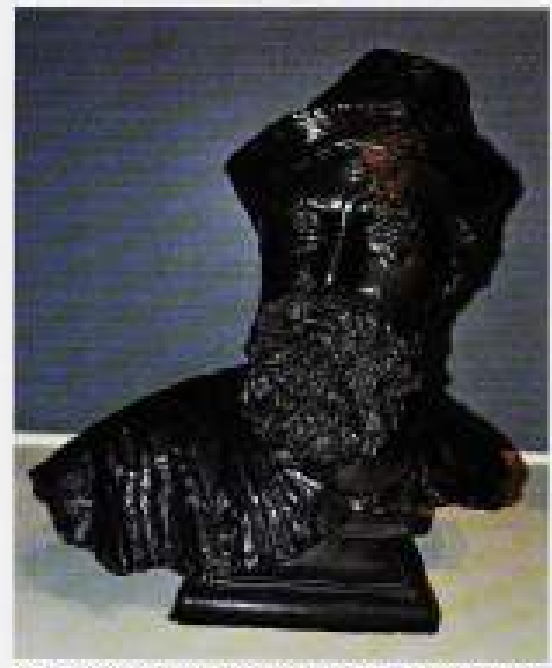

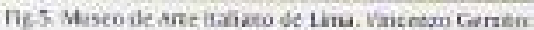

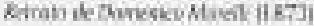

Yánnini Pareati resumió la casi frenérica actividad llevada a cabo buscando obras de arte en las mas importante ciudades italianas en un discurso pronunciado en abril de 1922 en ocasión de la Asamblea del Coanité Italiano que se estaba ocupando de la realizaciondel miseo en Lima." Un quehacer contimus con viajes a las diferentes oudades italiamas: Roma, Forencia. Venecia, Milãa. Trieste, Nápoles, etc. visitando las exposiciones públicas y las privadas organizadas en ese perioda, como la Primera Bienal Romana o la famosa exposición en la Galeria Pessiro de Milán para adgairir cuadros, escultuFas. obres graficas y objetos de arte decorativo de los artistas vivientes mas representativos de las escuelas pictóricas italianas, sia duda seáalados solure todo por Ojetti. No olvido a los artistas ligures, sicilianos, abruceses. to: dos presentes en fin para documentar, en $\mathrm{L}$ línea indicada por Ojetti, el caricter regional ded arte iraliano que se tenia que defender $y$ mostrar por su originalidad. Seria una injusticia mencionar a algunos artistas y no hablar de otros que, numerosos, colman con sus obras las salas del pabellón italiano de Lima; icomo no recordar, edemás de los arriba menciona: do5 Inrocenti e Canpaticit) (fig.2-3), por in mentos a los pintores Amando Spadini. Gulio Aristide Sartorio, Francesco Paolo Michetti. Plinio Nomellini, Antonio Mancini (fig.4). Adolfo De Carolis, Felice Carena, Carlo Follini, Ettore Tiso, Viacenzo liollire dY a los escoltores Vincenzo Gemito (fig.51, del cual el mu. see cantserva también un hermoso retrato ell sanguina, ${ }^{22}$ Libero Andreotti. Oreste Chilleri, Aurelio Mistruzzi, Leonardo Bistodf., Angeto Biancani. Enrico Tadolini. Pasquale Sgandurra, Domenico Irentacoste y muchos mas cuyas obras desde cast un siglo enriquecen el edificio italiamo del Parque Neptuno?

Hi moseo reune casi doscieztats coras de artistas nacidos todos en la segunda mitad del aigla XIX y que pertenecen a la tradición clasica italia. ha, con pocas excepciontes. No hay que olvidar. como ya serialó Quesada, que se clecifió no admitir a los artistas que yo habian fallecido o que

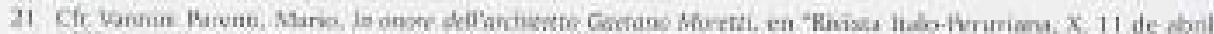

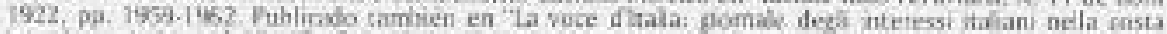

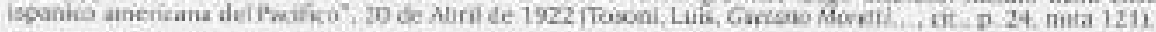

22 Qoesnia. Naris a carpa das Muvo d'Arte itainne ait po. 121-137. 
representaran las vanzuardias italianas cono el futurismo, la metafisica, el arte abstracto y el grupo relacionado con la revista Kubri Nestici. Como ya hemos indicade, el acto de ofre cer obras italianas al Perá era represeratacuón de cómo la tradición civilizadora del mensaje artistico italiano seguia con vida en ha prodocción artistica contemporänea. Fl proyecto del museo pensido por Vanaini Parenti y por Ojetn dejaba predecir un creamiento postenor y periódico de la colecoión que, at parte algana esporbdica adquisición, nunca se realizó."

\section{L.a inauguración del Museo de Arte taliano en tima}

Per fin el maseo se inauguró el 11 de noviembre de 1923, aniversario del nacimiento del rey de Italia Victor Emanuel Ill (11 noviembre 1869). Transcribo a continuación el texto de tua carta inedita de Mario Vamini Parenti enviada descle lima el 16 de noviembre de 1923 a Ugo Ojetti, para comunicarie la alegre noticia;

lima 16 de Novientre de 1923

Estimadísimo Siguor Ojetti.

1Mi hijo "el Peruano" por fin se inaugaról El Presidente, Ministros del Esta do. Dipkomáticos, Misiones Extranjeras y hasta el Cardenal Belloch y Vivú, arzobispo te Burgos, hidalgo tspanisal, y embajador de Su Santidad y con "carta del Rey Alfoasn de Fspaña", presenciaron la ceremonia. Me pernití telegrafiarle ya que se trataba de un acontecimiento de tamana importancia. Hasta se acufiam grandes aredallas de oro, lcuyo inayor valor es. su pese especificol part ofrecerlas an Monarcas, Minstros y personalidades de los dos paises. (No se ofenda pero también tengo una para Usted). La ceremonia fue magnifica, excepcional: el séquito con el que viajaba cl ilustre cardenal, ademas, genetó, por sus extraios uniformes al estilo de Pizarro, un efecto magnifico: parecia, IDios nos librel Un cuadro del Barbuda. Tengo que coxifesar que el Museo es una verdadera joye, donde hasta las obras mediocres lucen hermosas, El efesto moral sahre el pais y sohre los Ministros Plenipotenciarios. en especial los de las Potencias que son nuestras amigas queridas, fue colosal. Iralia, ademàs de ser el pais "de los trabajadores del Rey Victor Emanuel", se reconoció coino un pais de intelectuales, by ya era hors! iHay que dejar de creer en estos paises que cada iraliano con la cara limpia teaga que ser un inglés oun francés o qué sé yol (con resperto a esto siempre me acuerdo de cuánto the enoje de niño cuando, un día, una amiga de mi mamá isic) que me queria hacer un piropo, dijo: iqué lindo tu hijo parece extrajerol). (...). Entonces su carta que me llego aqui en lima. me ha llevido de alegria. la lei varias veces con placer: sus palabras de felicitación me compensaron de tanto trabajo, crè: me, sinceramente, y las ganas, que la inaugaración de nuestro Musco aqui fuera un verdadero evento que marcara la historia del Arte, laumentaron del trescientos por cientol Usted conocey sabe entusiasmar al corazon italiano, y muy frecuentemente una palabea dicha en el momento oportuno, hace más que cualquier recorpensa inientras que el contratiempo es th especialided de ciertos Nuestrosl Junto con su misivis, recibi tambën una carta de S.F. Centile que reemplazabs al telegrama que Usted anunció en el Corriere y que nunca llegó su parrafo parece haber puesto un énfasis

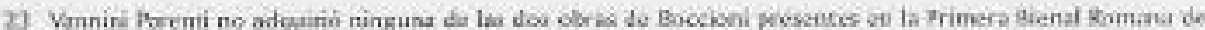

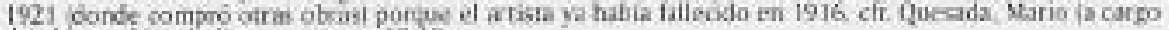

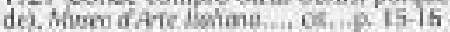

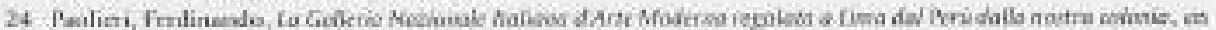
Ta Nurioset, Hootra, 9 de Eebreto de 1922 
mayer en la gratitud del Gobierno. Yo sigo con mi propaganda de Arte, sin parar frente a las pequecias emidias de personas que tienen problemsis de digestióa y de unos artistas que no merecen ni ser mencionados: es mi misión. Sin embatgo de manera un poxo soberbia me atrevo a dexir que en Sudamérica nuestro Arre Modemo ha vuelto a florecer y se encuentra en el primer lugar, y esto es suficiente para mi. De la coleccónt Carnielo vuelve casi todo a Italia, excepto unos diez bocetos, vendicos a tmos amigos coleccionistas, justo pora dejar aqui unos gemenes de Jos Macchiaioli. Creo que la enfermedad se va a propagar y que pronto veremos sus efectos. No tuve campo libre para hacer toda la publicidad que me habria gustado a la Exposición Retrospectiva, a causa de la envidia de artistas vivientes nuestros, que veian en eso una usurpacion. Me lo han dicho y escrito: y de hecho, para no crear discrepancies, expuse la colección en una sala del Museo, y no en el pabelión. Por la misma razón no aconsejé adquisiciones oficiales Su Fation, mire que gradoso, casi lo he vendido aqui en ef Perú. obviamente confiando en la palabra, al Comendador Gino Salocdai, Director del Banco Italiano, florentino, que viajará los primeros dias del año a Florencia. Fsta vez intentaré traer taenbeén el famoso "giacos" (sic). ya que mi amistad con el Señor Presiderte es miry buena: hasta nos otorgó. al Prof. Moretti y a mi, la orden suprema del Sol, una condecoracion excepcional, ide oro macizo y esmaltada! Una verdadera constelación. También le agradezco mucho lo que me comenta con respecto a la Exposicion de Paris estoy a su disposición en todo y para todo, feliz de tenerlo como jefe.

Mis respecos a la Señora Ojerti y a la Senorita tambien de parte de mi es. posal iNicoletta de verdad es muy divertidal Para Usted mis más cordiales y afectuosos saludos, Nario Vannini Parenti.

P.D. Le adjunto las direccicxes de algunos amigos que convenci para que se subscribieran a Dédalo, puede dar la orden de que se les envien la publicación y también la cuenta junto con el primer ejemplar: por fanot especiflque que ef recibo esté en liras italianas, porque se we feo que el cobro sea en francos franceses.

Yo viajo el 15 de Enero. ${ }^{3}$

El hijo "peruano" era, obviamente, el Museo de Arte Italiano de Lima y el orgulloso relato de la inauguración muestra de manera muy clara el cuidado y la pasión dedicados, a pesar de los obstáculos y las envicias, a la majestuosa y muy original iniciariva italiana en el Peri que valió a Gactano Moretti y a ell el gran honor de la Orden Suprema del SoL. El mismo Mannini Parenti le recordaba a Ojerti, en otra carta, del 31 de diciembre de 1922, "... Jas angustias y las ansias mal reconocidas" experimentadas durante su actividad de beisqueda de las obras para Lims, brisqueda que, por otro lado, Ojetti había descrito clogiándola en un articulo publicado en el "Cocriere della Sers" poce tiempo antes,"

Es interesante la referencia a la colección del escultor Rinalóo Camielo. Muchos cuadros de los Mecchinioir" estabun incluidos en ella y es evidente la intenciôn de Vannini Parenti de hacerlos conocer en Lima y motivar adqutisiciones de los mismos.

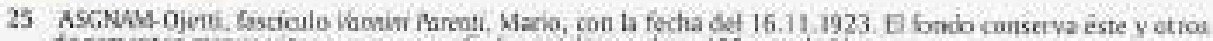

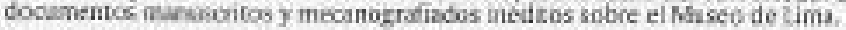

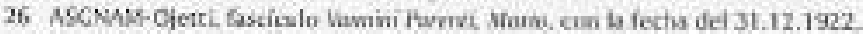

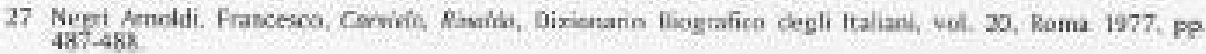


En el misms fondo de archiwo se conserva una copia de un largo articulo de "Lia Prensa" que se publicó el mismo dia de la inauguracion, con las fotos del museo, de sus interiores y una descripcion del edificio y de las salas, y dos grandes fotografias con los retratos tel Rey y del Primer Ministro Bento Mussolini (tig.6).

\section{Ei Musen de Arte ltaliane boy}

Lamentablemente, sólo diez años después de la inauguración. el Museo de Arte Italiano de Lima se encontraba en condiciones precarias. Dos cartas enviadas en enero de 1933 a Oetti por Vannini Parentiy por Gino Salocchi respectivamente ique seguramente habian sido coordaradas), hacen manifiesta la situacion de crisis de la instinucien:

Forencia 30.1.33

Mury Estimado Seño: Ojetti.

Me causa un gran dolor que el Gobierno del Perù no se preocupe por el deterioro de las obras y de los locales de la Galeria de Arte Italiano de Lima. Nuestra Cobonia renia la intencion de adquirit las obras de nuevos artistas recién aparecidos dutante esta decada que va de la inauguración de la Galenia hasta el dia de hoy. La actitud de las autoridades Peruanas ha desanimado nuestra moble iniciativa, $y$ de esta nanera la Galeria piercle el objetivo màs importante por el que se creó: es decir enseñar, con continuas y oportunas adquisiciones, las tendencias artisticas de nuestro país. Tambien pienso que una critica suya hacia esta forma de actuse podra motivar a quiénes deberian preocuparse por la conservación de una obra |sic| tas importante en el ambito artistico y en el ámbito politico, siendo la Colonia Italianta la mas numerosa del Perú. Yo tambien le agradezco mucho todo ko que pueda hacer y le envio cordiales saludos. Vanmini.

Bnos dias antes también Gino Salocchi le habia escrito a Ojetti:

Lima 26 de Enero de 1933

Su Excelencia.

Orazio Pedrazzi, Embajador de Italia en Cuile, me escribio para pasarme la copia de tuna carta suya para Usted referente a la Galeria de Arte Maderno de Lima. É, que es muy amable conmigo en dicha carta. reitera la petición que vo le hice personalmente, es decir de no olvidar en su "Décalo" el valor untrínseco y moral que cida dia mís adquiete la colección de arte de lima desafortunadamente abandonada. De heche nosptros los italianos de aca tenemos que acercarnos al tema con clerta diplomacia ya que se trana de un regalo que hemos hectio. En los ültimos tiempos ha habido infinitos cambios en el Ministerio de la Edacación Pública por los tumultos politicus, así que es ficil comprender que el Gobiemo Peruano no preste toda la atención que deberia a la conservación de la Galería. Cuanda tuvimos la oportunidad de ofrecer la restauracion, las personas a las cuales nos hemos dirigido no quisieron aceptar atestra oferta porque consideraron que en tarea de ellos mismos reparar los daños del edificio y mantener ordenada las obras. Lo que quiero decir es que las autorichades demuestran periectamente la compreasión moral de la situacion: pere lamentablemente nodie ha actuade y las cosas siguea como S.E. Redrazis se las describio. Yo me atrevo a intervenir porque conozco Su

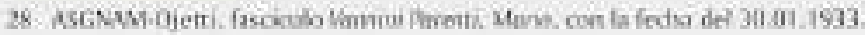


amabilidad y sé que Usted me permite ocuparme de este tema tan delicado. Confio además en las acciones que Usted teadria la curtesia de llevar a cabo. que tendrin una importancia decisiva por el alto significado de Su palabra ilustre y por la pertinencia con la que Usted siempre trata todos los argumentos. 1.... Gino Salocchi. ${ }^{n}$

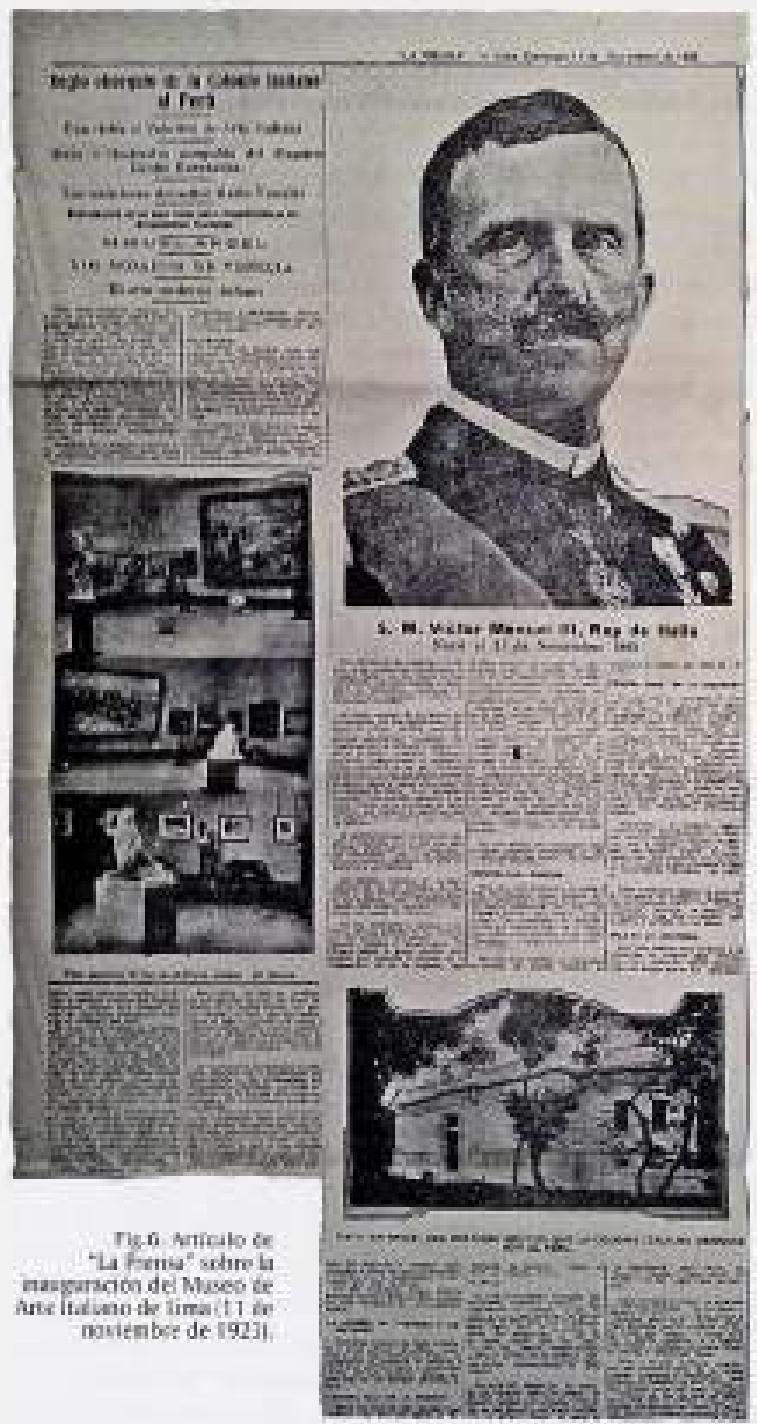

Las rasivas exponian un pro. blema real oon respecto a la situación del nuevo museo, creas do por la comunidad italiana pero ya pasado a ser propiedad del gobiemo peruano, que, por ende, tenia que administrarlo. Se entiende sobre todo por parte de Sakcchi el aridado y, de alguna manera, la comprensiox con respecto a la situacion poütica peruana de esos anos que ispedia prestar atención a un problema, si importante, pero no priocitario. Es evidente cuanto el famoso critico italiarno sea terido en consideracion por Varami Parenti y Salocchi y también por el embaja dot de fralia en Chale, Orazio Pedrazzi, que creian que si el bubiese publicado un articulo sobre la cuestion del Musea de Arte Italiano de lima en la (revista) "Dédalo" habria podich en cierta medida untluir positivamente.

Eil Museo de Arte Ltaliano de Lima se quedó sin embargo practicamente en las mismas condiciones bajo la administra. ción de la colonia italiana hasta el aiso 1972, cuardo fue oficial. mente entregado al Instituto Nacional de Cultura del Perú. Las pocas adquisicicoes suce. sivas que se hicieron se docu. mentaron en un nuevo catálogo publicado en 1938.

29 Wisen fasciculo Sobachi cho cos la fecta ale 26.01 1973

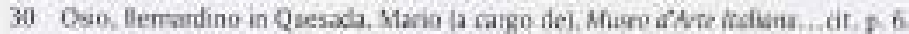

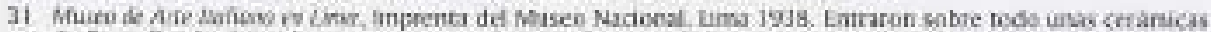

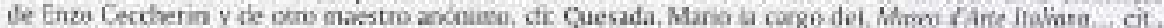

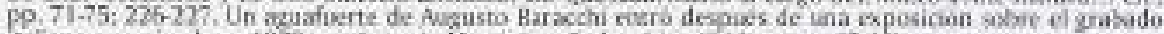

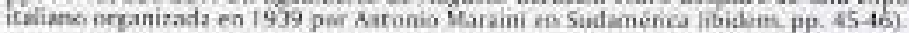


Por lo menos desde 1975 hasta hoy esta documentada una intensa y continua actividad de exposiciones temporales en los espacios del museo. las cuales estain descritas en un informe que muy amablemente me facilito la actual directora, la doctora Margarita Cinocchio Lainez Lozada. En el sc detallan exposiciones de diferentes tipos, de las monograficas sobre artistas contemporalteos, tambient extranjeros, a otrats solve artesantia, diseno, etc. altemedas con exposiciones de la colección perananente del museo (Colección Museo de Arte Italiano) y también concierros. "Fl único ano que faltze en la tista es el de 1990, quizás porcue se estaba preparando el catálogo de la adquisición de muevas obras de veiatiséts artistas italiaros donadas al Museo de Arte tealiano de lima, gracias al interés demostrado por el arriba tnencionado embajador Bernardino Osio. Fue el primer acto del embajador de Italia en el Perú Bemardino Osio después de visitar el museo en 1990 y enconcrar el edificio y las obras en un estado de triste abandono. Situación ya totalmente cambiada y mejorada, gracias al mismo embajador que constituryo la Ascociación de los Amigos del Vusco de Arte ltaliano cuyos inregrantes son itals-peruanos y cuyo actual presidente es Gian Havio Gerbolini Isola. La asociación en seguida se preocupó por encontrar los recur. sos para semodelar el museo danado a causa de la explosión de un coche bomba ef 7 de febrero de 1992 y por planear, coms ya hemos dicho, la redacción de un muevo catalogo cientifico de la colección, encargado al profesor Mario Quesada, quien, con su grupo de alumnos, realizó une impecable publicación ilustrada de tadas las obras de propiedad de musen.14

Ahora Wiendo la renovada e intensa actividad del Museo de Arte ltatiano de Lima me gustaria terminar mi contribuciōn retomando el proyecto de una exposición en lima y en Roma. sobre la historia del museo de la comunidad italiana, que no se logró hacer en 2011. Seria un imprescindible acto de reconocimiento de un episodio tan peculiar de la historia de las museos y del coleccionismo italiano y peruanol Itahiano de Lima. Lima 1990. Et catálogo muestra las obras de las artistas italianos adecaridas despues Ce les

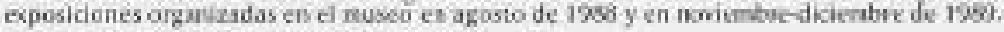

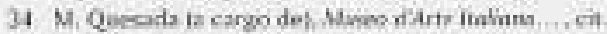

